\title{
EDITORIAL
}

\section{Introducing a new role for BNP: as a general indicator of cardiac structural disease rather than a specific indicator of systolic dysfunction only}

\section{A D Struthers}

Could BNP be a good prescreening test for echocardiography in general rather than a specific test to prescreen for left ventricular systolic dysfunction only?

1 n the early 1990s, the idea first arose that plasma concentrations of $\mathrm{B}$ type natriuretic peptide (BNP) might help target scarce cardiac investigations towards the most deserving. ${ }^{1}$ This mainly referred to the possibility that BNP could detect which patients had heart failure and which did not. This was based on the fact that echocardiography slots were limited, and that heart failure was hard to diagnose accurately on clinical grounds. The need for correct targeting of echocardiography increased when it was fully realised that clinical heart failure was a heterogeneous entity ${ }^{2}$ and that the treatments which produced major benefit (angiotensin converting enzyme (ACE) inhibitors and $\beta$ blockers) were only of definite benefit in those with left ventricular (LV) systolic dysfunction.

A huge amount of work then went in to establishing how well BNP identified either heart failure or LV systolic dysfunction. ${ }^{34}$ The bottom line of this work is that a normal BNP is a very effective way of "ruling out" heart failure or LV systolic dysfunction in either a symptomatic or an asymptomatic patient. In this way, echocardiography could be reserved only for those with a high BNP concentration. However, it became patently clear that a high BNP in itself did not always indicate LV systolic dysfunction. Thus for diagnostic purposes BNP was sensitive but not specific-that is, although a high BNP was picking up nearly all LV systolic dysfunction, it appeared to also pick up some other patients. This begs the obvious question: what other patients were being identified by a high BNP concentration?

\section{A PROGNOSTIC INDICATOR}

Another common finding with BNP was that BNP was much better as a prognostic indicator than it was as a diagnostic indicator. ${ }^{5}$ This was even the case in an elderly free living population in the community. ${ }^{6}$ This was important because it raised the idea that the patients who had a high BNP but had normal LV systolic dysfunction had a non-innocent reason for this high BNP concentration, which again adds impetus to the question of what else BNP was picking up apart from LV systolic dysfunction.
Related to this is the obvious point that echocardiography is not done only to pick up LV systolic dysfunction. Rather it is used to pick up valve disease, left ventricular hypertrophy, and diastolic dysfunction, among others. Therefore, could it be that BNP would be a good prescreening test for echocardiography in general rather than a specific test to prescreen for LV systolic dysfunction only?

In fact, such a prospect seems very likely since BNP is really an indicator of raised intracardiac pressure, irrespective of whether the raised intracardiac pressure is caused by LV hypertrophy (LVH), LV systolic dysfunction, valve disease or even fast atrial fibrillation. The paper by Nakamura and colleagues ${ }^{7}$ in this issue shows that this is indeed the case. In fact, previous work had already suggested that a high BNP was highly accurate in detecting LVH in hypertensives. ${ }^{8} \quad$ Nakamara and colleagues ${ }^{7}$ screened around 1100 subjects and identified heart disease in $4.3 \%$. The underlying cardiac diseases were heterogeneous including atrial fibrillation, hypertensive heart disease, and valvar heart disease. The numbers with each separate cardiac disease were small. Nevertheless, the sensitivity and specificity of BNP for each of these cardiac diseases was 100\% sensitive and $83-97 \%$ specific. Only angina was poorly detected by BNP, which is not surprising since angina alone will not increase an individual's intracardiac pressure at rest. Indeed, Nakamura and colleagues ${ }^{7}$ speculate very reasonably that in the papers in which BNP did not perform well at identifying LV systolic dysfunction, this was probably because the population studied was contaminated by the other cardiac diseases which also increased BNP. As a footnote, it is intriguing that in a Japanese population study of over 1000 patients, with a mean age of 56 years, not one single patient had an LV ejection fraction $<40 \%$. This low frequency clearly differs from western societies with more ischaemic heart disease. $^{4}$

\section{"BNP is really an indicator of raised intracardiac pressure"}

In a sense, it is surprising that BNP researchers focused for so long on the single issue of whether

Abbreviations: $A C E$, angiotensin converting enzyme; BNP, B type natriuretic peptide; LV, left ventricular; LVH, left ventricular hypertrophy. 
BNP identified LV systolic dysfunction or not, and did not recognise that BNP could be used in a more general way to select patients for all echocardiography in order to detect not only LV systolic dysfunction but also LVH, LV diastolic dysfunction, atrial fibrillation, and significant valve disease.

\section{BNP AND LVH}

Clearly more work is required to justify this idea but it could give BNP a wider role than has hitherto been suggested. One of the wider roles which would be particularly helpful relates to LVH. In olden days, it was not thought crucial to detect which hypertensives (or diabetics) had echo LVH or not because it was not certain that LVH regression was beneficial. However, the data of Verdecchia and colleagues ${ }^{9}$ changed all that when he showed that regressing LVH virtually quartered the risk of future cardiovascular events and that blood pressure in itself was not a sufficient guide that LVH had regressed. Ensuring LVH is regressing may be important not only for hypertensives: it may also be relevant to diabetics who have a surprisingly high incidence of $\mathrm{LVH}$, including normotensive diabetics. ${ }^{10}$ Furthermore, ACE inhibitors are no magical panacea for LVH. ${ }^{11}$ It could turn out that the correct target blood pressure in a patient with LVH is whatever blood pressure reduces LVH in that individual, rather than a global generic target blood pressure for all. This even raises the idea of using BNP serially to ensure that LVH is indeed regressing in an individual patient.

In conclusion, BNP could become a useful test to screen for significant valve disease, LVH, atrial fibrillation, and LV diastolic dysfunction as well as its more traditional role in screening for LV systolic dysfunction. In this way, it could become a general screening test for all echo requests, irrespective of whether the echo request is to look for the causes of symptoms (when the echo request is looking for valve disease or LV dysfunction), or whether the echo is being done as a screening procedure to look for asymptomatic killers such as LVH.

\section{REFERENCES}

1 Struthers AD. Plasma concentrations of BNP: will this new test reduce the need for cardiac investigations? Br Heart J 1993;70:397-8.

2 Struthers AD. The diagnosis of heart failure. Heart 2000;84:334-8.

3 Cowie MR, Struthers AD, Wood DA, et al. The value of natriuretic peptides in assessment of patients with possible new heart failure in primary care. Lancet 1997;350:1349-53.

4 McDonagh TA, Robb SD, Murdoch DR, et al. Biochemical detection of IV systolic dysfunction. Lancet 1998;351:9-13.

5 McDonagh TA, Cunningham DA, Morrison CE, et al. LV dysfunction, natriuretic peptides and mortality in an urban population. Heart 2001;86:21-6

6 Wallen T, Landahl S, Hedner T, et al. BNP predicts mortality in the elderly. Heart 1997:77:264-7.

7 Nakamura $M$, Endo $H$, Nasu $M$, et al. Value of plasma B type natriuretic peptide measurement for heart disease screening in a Japanese population. Heart 2002;87:131-5.

8 Kohno $M$, Hario T, Yoko Kawa K, et al. BNP as a cardiac hormone in essential hypertension. Am J Med 1992:92.29-35.

9 Verdecchia P, Schillaci G, Bragioni C, et al. Prognostic significance of serial changes in LV mass in essential hypertension. Circulation 1998;97:48-54.

10 Devereux RB, Roman M, Paranicas M, et al. Impact of diabetes on cardiac structure and function. The strong heart study. Circulation 2000;101:2271-6.

11 Liebson PR, Grandits GA, Dianzumba S, et al. Comparison of five antihypertensive monotherapies and placebo for changes in LV mass in patients receiving nutritional-hygienic therapy in the treatment of mild hypertension study (TOMHS). Circulation 1995;91:698-706.

\section{IMAGES IN CARDIOLOGY}

\section{Contrast enhanced magnetic resonance angiography of severe aortic coarctation}

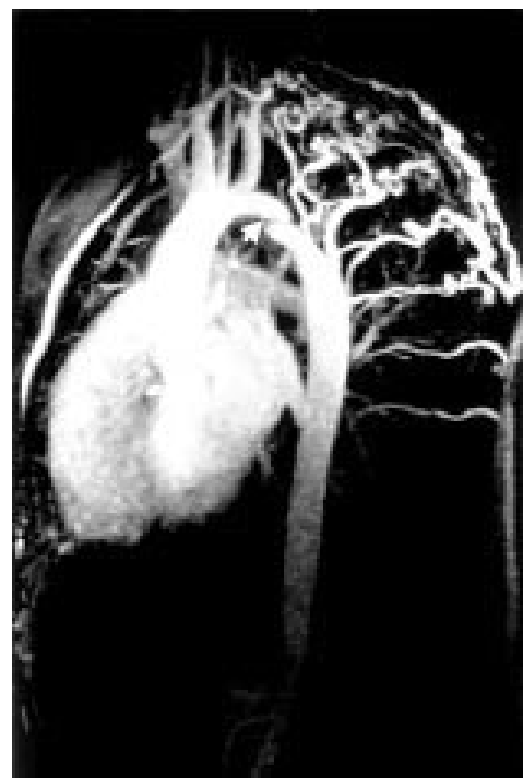

A previously healthy 21 year old man first presented with subarachnoid haemorrhage secondary to a right posterior communicating artery aneurysm, which was surgically clipped. He was discovered to be hypertensive, and clinical signs were suggestive of aortic coarctation. Echocardiography confirmed the presence of a severe coarctation. Continuous wave Doppler interrogation of the descending aorta from the suprasternal approach recorded a peak velocity of $4.4 \mathrm{~m} / \mathrm{s}$ and significant diastolic forward flow. However, the anatomy around the coarctation was not well defined because of tortuosity of the coarctation segment. Contrast enhanced magnetic resonance angiography (CE-MRA) was requested before surgical intervention. The presence of a Sugita brain aneurysm clip (which is made of a cobalt chrome alloy) did not contraindicate CE-MRA - a fact confirmed by consulting both the manufacturer and available safety databases. Maximal intensity projection image of CE-MRA in the left anterior oblique view (left) revealed a focal tight (diameter $6 \mathrm{~mm}$ ) juxtaductal coarctation (arrow), immediately distal to the origin of the left subclavian artery. An extensive network of enlarged and tortuous collateralising intercostal and internal mammary arteries is evident. The patient was rescheduled for early operative repair of the severe coarctation. 